\title{
Effect of glyceryl trinitrate on the sphincter of Oddi motility and baseline pressure
}

\author{
M STARITZ, T PORALLA, K EWE, K-H MEYER ZUM BÜSCHENFELDE \\ From the 1st Department of Internal Medicine, Johannes Gutenberg University of Mainz, Mainz, Federal \\ Republic of Germany
}

\begin{abstract}
SUMmARY It is widely accepted that glyceryl trinitrate (GTN) effectively dilates the smooth muscles of blood vessels. A similar effect has been postulated on the smooth muscles in the gastrointestinal tract. In this study the motility of the sphincter of Oddi and the common bile duct pressure as determined by endoscopic manometry was investigated in nine patients before and after sublingual application of $1.2 \mathrm{mg}$ GTN (nitro group). Eight untreated patients served as controls. Three minutes after application of GTN the papillary contraction amplitude decreased from $69.3 \pm 4.3 \mathrm{mmHg}$ to $36.8 \pm 5 \cdot 1 \mathrm{mmHg}(\mathrm{p}<0.005)$ and the papillary baseline pressure fell from $8.9 \pm 0.6 \mathrm{mmHg}$ to $2.9 \pm 0.2 \mathrm{mmHg}(\mathrm{p}<0.005)$ respectively. The contraction frequency in the nitro group and all motility parameters in the control group remained unchanged. These results indicate that GTN does not influence the sphincter of Oddi motility, but it relaxes very effectively the sphincter of Oddi muscle. Thus, GTN should be taken into account for the treatment of biliary colic. In our endoscopic unit GTN proved to be useful as premedication for endoscopic examinations, particularly for the removal of small and medium size common bile duct stones through the intact papilla.
\end{abstract}

The sphincter of Oddi is the smooth muscle choledochoduodenal junction providing regulation of bile flow and hindering duodenobiliary reflux. The effect of ethanol on the sphincter muscle has narcotics ${ }^{1-3}$ on its function has been estimated by indirect measurement of its flow resistance using the approach via T-drainage intra- or postoperatively. The effect of ethanol on the sphincter muscle has recently been determined directly by endoscopic manometry. ${ }^{4}$ This procedure permits direct recording and quantification of the sphincter of Oddi motility its baseline pressure and the common bile duct pressure without further molestation of the patient during ERCP examination. The sphincter motility is described by the papillary contraction frequency (contractions per minute) and the contraction amplitude. During sphincter contractions the papilla is closed and bile flow stops. Between these contractions the papillary muscle is relaxed. Its baseline pressure mirrors the resistance

Address for correspondence: Dr M Staritz, 1 Medizinische Klinik und Poliklinik, Langenbeckstraße 1, D 6500 . Mainz, FRG.

Received for publication 4 May 1984 against bile flow which is propelled by the pressure gradient between common bile duct and duodenum (common bile duct pressure). ${ }^{5}$

Glyceryl trinitrate (GTN) is an organic nitrate which has been shown to relax the smooth muscle of blood vessels. This effect has been widely accepted for the treatment of angina pectoris. The following study was designed to evaluate whether GTN provides dilatation of the smooth sphincter of Oddi (SO) muscle. The effect of GTN on the SO motility, its baseline pressure, and the common bile duct pressure was investigated in nine patients, Eight untreated patients served as controls.

\section{Methods}

PATIENTS AND STUDY DESIGN

Seventeen patients who underwent endoscopic retrograde cholangiopancreaticography (ERCP) in order to rule out pancreaticobiliary disease were included. The endoscopic manometry was performed before ERCP in all cases. All patients showed completely normal results of ERCP and ulcers of the stomach or duodenum, oesophageal reflux, diabetes mellitus, and systemic diseases were 
excluded by appropriate methods. Alcoholism and drugs affecting gastrointestinal motility were anamnestically ruled out. From all patients informed consent was obtained. They were divided into two groups:

\section{NITRO GROUP}

In nine patients (five men, four women, mean age 43 years, range 18-62 years) the SO baseline motility, the baseline pressure and the common bile duct pressure were obtained before $1.2 \mathrm{mg}$ glyceryl trinitrate (Nitrolingualspray, Pohl-Boskamp, Hohenlockstedt, W Germany) were sprayed onto the tongues of the patients. Three minutes later endoscopic manometry was repeated.

CONTROL GROUP

An equal manometric procedure without GTN applications was performed in a control group consisting of eight patients (five men, three women, mean age 40 years, range $22-52$ years).

\section{ENDOSCOPIC MANOMETRY}

After a 12 hour fasting period and premedication with $10-15 \mathrm{mg}$ diazepam which had been shown by $\mathrm{Nebel}^{7}$ and in an own pilot study not to affect SO motility, the papilla of Vater was intubated with a triple lumen catheter using a sideview endoscope and standard ERCP technique (endoscope Olympus JF $1 \mathrm{~T}$ ). All manometries were obtained in left lateral side position of the patients. The triple lumen catheter (Medi-tech, Watertown, USA) had a length of $200 \mathrm{~cm}$, a diameter of $1.7 \mathrm{~mm}$ and a luminal diameter of $0.5 \mathrm{~mm}$. The lumina ended as sideholes at the tip of the probe. The length of the intubation into the $\mathrm{SO}$ could be estimated by a ring marking of the distal catheter end. The correct catheter position in the common bile duct was confirmed by gentle aspiration of bile through one of the lumina. Two of the three lumina were perfused by bubble free saline at a rate of $0.2 \mathrm{ml} / \mathrm{min}$ by the hydraulic capillary perfusion pump according to Arndorfer. ${ }^{8}$ The catheter was attached to two Statham elements (Beckman, type R 427 G). The recorded pressure was registered by a writer (Beckman R $511 \mathrm{~A}$ ) at a paper running speed of 1 $\mathrm{mm} / \mathrm{sec}$.

At the beginning of each manometric recording the triple lumen catheter was introduced into the common bile duct for registration of its pressure. Thereafter the papillary motility was recorded for approximately three minutes. The duodenal pressure was obtained positioning the manometry catheter free in the duodenal lumen at the site of the papilla. For scoring the manometric tracings the sphincter residual pressure was plotted against the common bile duct pressure and the latter against the duodenal pressure taken as zero-reference (Fig.1). The sphincter contraction amplitude was plotted against the sphincter baseline pressure. The sphincter contraction frequency and the amplitude were calculated as mean values out of 10 contractions.

Values are reported as mean $(\overline{\mathrm{x}}) \pm \mathrm{SEM}$. For statistical analysis the Wilcoxon's test was used.

\section{Results}

The basal values recorded for the papillary contraction frequency, the contraction amplitude, the papillary baseline pressure, and the common bile duct pressure were nearly identical in the nitro group and in the control group respectively (Figs. 2-5).

In the nitro group the contraction frequency before and after nitro application revealed no difference with $6 \cdot 0 \pm 0 \cdot 1$ and $6 \cdot 1 \pm 0 \cdot 1$ contractions per minute respectively (Fig. 2). The contraction amplitude, however, decreased from $69 \cdot 3 \pm 4 \cdot 3$ $\mathrm{mmHg}$ to $36 \cdot 8 \pm 5 \cdot 1 \mathrm{mmHg}(\mathrm{p}<0 \cdot 005$, Fig. 3 ). The papillary baseline pressure significantly decreased from $8.9 \pm 0.6 \mathrm{mmHg}$ to $2.9 \pm 0.7 \mathrm{mmHg}(\mathrm{p}<0.005$, Fig. 4). The common bile duct pressure showed a small decrease from $8 \cdot 4 \pm 0.7 \mathrm{mmHg}$ to $7 \cdot 1 \pm 0.6$ $\mathrm{mmHg}(\mathrm{p}=0.05$, Fig. 5). The manometric examinations of the control group, however, revealed nearly identical results concerning the contraction frequency, the contraction amplitude, the papillary
Fig. 1 Endoscopic papillary and bile ducts manometry. Typical localisation of the manometry catheter in the sphincter of Oddi segment, the common bile duct and the duodenal lumen.

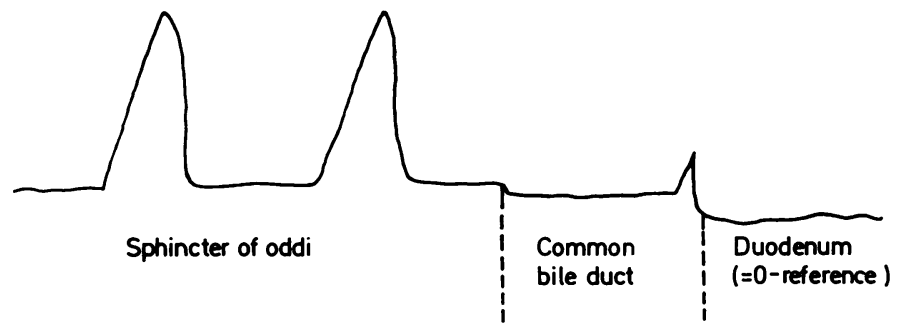




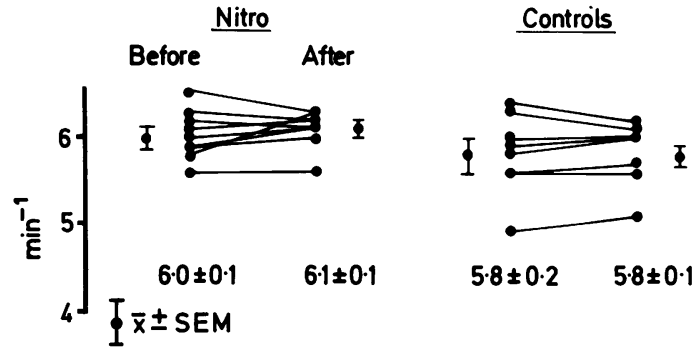

Fig. 2 Papillary contraction frequency before and after application of GTN compared with controls.

baseline pressure, and the bile duct pressure (Figs. $2-5)$. There were no complications in any patient after endoscopic manometry.

\section{Discussion}

Our data indicate that glyceryl trinitrate (GTN) markedly and significantly affect the sphincter of Oddi muscle. While its motor activity described by the contraction frequency remained unchanged, its muscle tone fell to approximately $30 \%$ of its normal values as the assessment of the papillary contraction amplitudes and the SO baseline pressure demonstrated. In this way the papillary lumen was

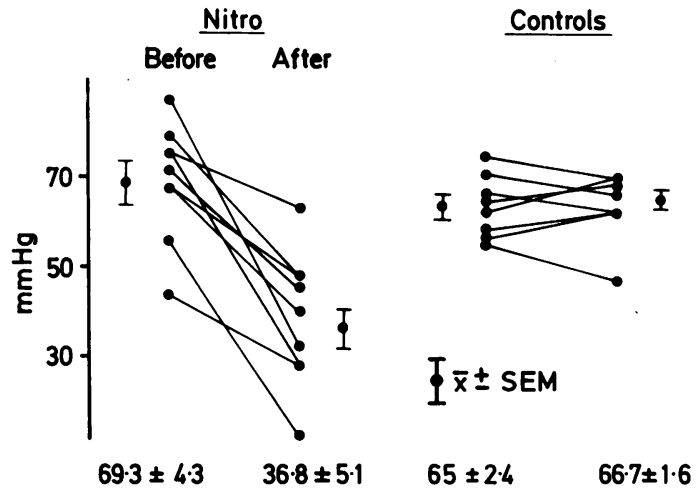

Fig. 3 Papillary contraction amplitude before and after application of GTN.

'widened'. The common bile duct pressure serves as indicator for the papillary function ${ }^{1-3}$. Glyceryl trinitrate application diminished the bile duct pressure. This indicates that the drug reduces the resistance to the bile flow.

The results of our research conflict with findings reported by Meckeler and Borow. ${ }^{9}$ The authors did not find the presumed dilating effect of GTN. Their study, however, has been performed on patients eight days after cholecystectomy. We, therefore, consider, that the unphysiologic conditions explain the conflicting results.

The sphincter dilatating effect of GTN served as premedication for ERCP examinations. Compared

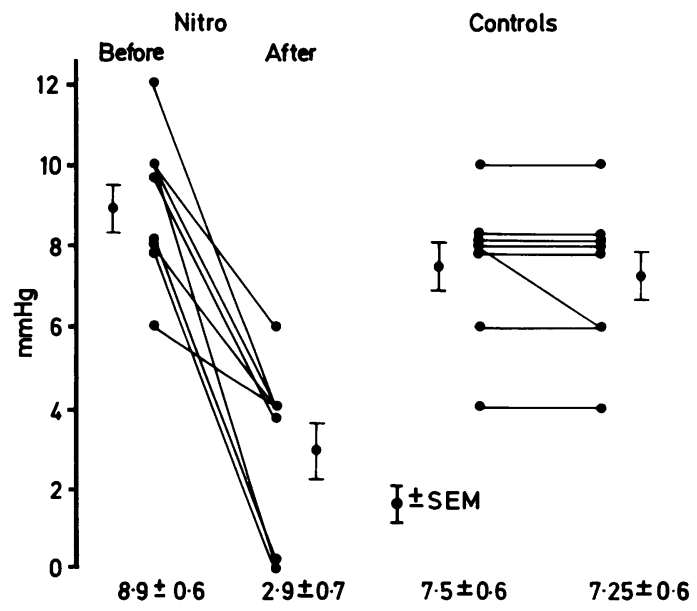

Fig. 4 Papillary baseline pressure before and after application of GTN.

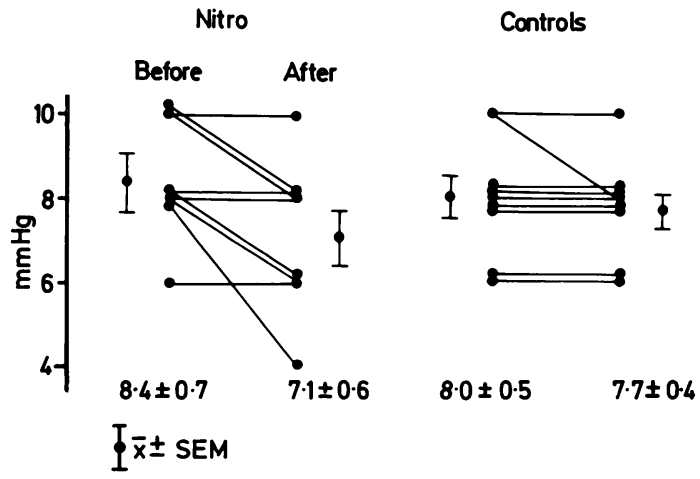

Fig. 5 Common bile duct pressure obtained before and after application of GTN. 
with the failure rate of ERCP amounting to $5 \%{ }^{11}$ at least in general - in our endoscopic unit during a seven month period with GTN premedication not a single ERCP out of 450 examinations failed because of papillary intubation problems.

The greatest progress resulting from GTN application, however, was the removal of small and medium sized bile duct stones through the intact papilla without endoscopic papillary dilatation ${ }^{12}$ or even endoscopic papillotomy. ${ }^{13}$

The endoscopic manometry has verified the presumed dilating effect of GTN on the sphincter of Oddi muscle. The duration of this effect could not be determined exactly. Our experience during endoscopic procedures (removal of bile duct stones) indicates a duration of the described effect of approximately 15 minutes. This corresponds to the blood concentrations of GTN. After lingual application maximum blood concentrations appear within 2 minutes. It disappears on a short half-life time of 5 minutes. ${ }^{14}$ According to our data the sublingual administration is sufficient for endoscopic indications and for the application during biliary colic. For chronic therapy long acting nitrates should be administered.

\section{References}

1 Greenstein AJ, Kaynan A, Singer A, Dreiling DA. A comparative study of pentazocine and meperidine on the biliary passage pressure. Am J Gastroenterol 1972; 58: $417-27$.

2 Tigerstedt I, Turunen M, Tammisto T, Hästbacka J. The effect of Buprenorphine and Oxycodone on the intracholedochal passaage pressure. Acta Anaesthiol Scand 1981; 25: 99-102.

3 Hopton DS, Torrance HB. Action of various analgesic drugs on the human common bile duct. Gut 1967; 8: 296-300

4 Viceconte G. Effects of ethanol on the sphincter of Oddi: An endoscopic manometric study. Gut 1983; 24: 20-7.

5 Geenen JE, Hogan WJ, Dodds WJ, Stewart ET, Arndorfer RC. Intraluminal pressure recording from the human sphincter of Oddi. Gastroenterology 1980; 78: $317-24$.

6 Winsor T, Berger HJ. Oral nitroglycerin as prophylactic anginal drug: Clinical, physiologic, and statistical evidence of efficacy based on a three-phase experimental design. Am Heart J 1975; 90: 611-26.

7 Nebel OT. Manometric evaluation of the papilla of Vater. Gastrointest Endosc 1975; 21: 126-8.

8 Arndorfer RC, Steff JJ, Dodds WJ, Linehan JH, Hogan WJ. Improved infusion system for intraluminal oesophageal manometry. Gastroenterology 1977; 24: 7-23.

9 Meckeler KJH, Borow M. Direct manometric study of the sphincter of Oddi in man Gastroenterology [Abstract] 1975; 68: 1038.

10 Brandstätter $\mathrm{G}$. Pharmacological pressure reduction in the human common bile duct. Z Gastroenterol 1983; 21: $168-74$.

11 Kasugai T. Recent advance in the endoscopic cholangiopancreaticography. Digestion 1976; 13: 7681.

12 Staritz M, Ewe K, Meyer zum Büschenfelde KH. Endoscopic papillary dilation (EPD) for the treatment of common bile duct stones and papillary stenosis. Endoscopy 1983; 15: 197-8.

13 Safrany L. Duodenoscopic sphincterotomy and gallstone removal. Gastroenterology 1977; 72: 338-42.

14 Armstrong PW, Armstrong JA, Marks GS. Blood levels after sublingual nitroglycerin. Circulation 1979; 59: $585-8$. 